\title{
Nano-Screws: Asymmetrical Etching of Silver Nanowires
}

Rachel Lee Siew Tan, ${ }^{\ddagger, \S}$ Wen Han Chong, ${ }^{\ddagger}$ Yuhua Feng, ${ }^{\ddagger}$ Xiaohui Song, ${ }^{\ddagger}$ Chu Long Tham, ${ }^{\S}$

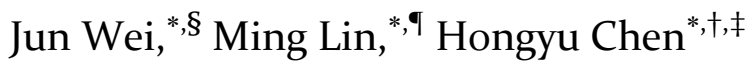

${ }^{\dagger}$ Institute of Advanced Synthesis, School of Chemistry and Molecular Engineering, Jiangsu National Synergetic Innovation Center for Advanced Materials, Nanjing Tech University, Nanjing 211816, China

${ }^{\ddagger}$ Division of Chemistry and Biological Chemistry, School of Physical and Mathematical Sciences, Nanyang Technological University, Singapore 637371

${ }^{\S}$ Singapore Institute of Manufacturing Technology, Agency for Science, Technology and Research (A*STAR), Singapore 637662

IInstitute of Materials Research and Engineering, Agency for Science, Technology and Research (A*STAR), Singapore 138634

\section{Materials}

Silver nitrate $\left(\mathrm{AgNO}_{3}\right), 99.0 \%$ (209139); poly(vinylpyrrolidone) $\left(\mathrm{C}_{6} \mathrm{H}_{9} \mathrm{NO}\right)_{\mathrm{n}}, \mathrm{PVP}, \mathrm{Mw} \sim 55000$ (856568); copper chloride dihydrate $\left(\mathrm{CuCl}_{2} \cdot 2 \mathrm{H}_{2} \mathrm{O}\right), 99.999 \%$ (459097); (3-aminopropyl)triethoxysilane (APTES) were purchased from Sigma Aldrich. Ethylene glycol $\left(\mathrm{C}_{2} \mathrm{H}_{6} \mathrm{O}_{2}\right)$, 99\% (9300-03) was purchased from J. T. Baker. All reagents were used without further purification.

\section{Characterization}

Transmission electron microscopy (TEM) images were obtained with a JEM-1400 (JEOL) operated at $100 \mathrm{kV}$. Field emission scanning electron microscopy (SEM) images were acquired from a JEOL JSM-6700F. Structural analysis and 3D electron tomography of nanowires were performed on an FEI Titan80/300 S/TEM (Scanning/Transmission Electron Microscope) (200 keV), which is equipped with a High-Angle Annular Dark-Field (HAADF) detector, a monochromator and a single-tilt tomography holder.

\section{D Electron Tomography}

A total of 76 HAADF-STEM images for STEM tomography were collected over a tilt range of $-74^{\circ}$ to $76^{\circ}$, with $2^{\circ}$ tilt steps. The stage tilting and image acquisition were done automatically by FEI Xplore-3D tomography software, while the tracking and refocusing of the area of interest were carried out manually in order to shorten the total acquisition time, minimize the beam damage to the sample and reduce the build-up of contaminants under prolonged beam irradiation. The magnification was 89,000 and 44,000 times respectively, corresponding to 1.53 and $2.96 \mathrm{~nm}$ per pixel. The acquisition time for one $1024 \times 1024$ sized image is $30 \mathrm{~s}$. The final tilt series data was aligned using 
cross-correlation method and reconstructed by Simultaneous Iterative Reconstruction Technique (SIRT, 50 iterations) of Inspect3D software, and the reconstructed 3D volume was visualized by surface and voxel rendering using Amira 4.1.

\section{Cross Section of Ag Nanowires}

Cross section TEM samples were prepared by dispersion of Ag nanowires in epoxy (Specifix 20, Struers) and cut by microtome.

\section{Synthesis of Thin Ag Nanowires}

For a typical synthesis, $25 \mathrm{~mL}$ of ethylene glycol was added to a $100 \mathrm{~mL}$ round bottom flask with a stir bar added. The round bottom flask was then suspended in an oil bath with thermostat set at $151{ }^{\circ} \mathrm{C}$ and heating for $1 \mathrm{~h}$ under magnetic stirring $(250 \mathrm{rpm})$. All reagents were dissolved in ethylene glycol. Then, $200 \mu \mathrm{L} \mathrm{of} \mathrm{CuCl}_{2} .2 \mathrm{H}_{2} \mathrm{O}(4 \mathrm{mM})$ was added into the flask and allowed to heat for another $15 \mathrm{~min} .7 .5 \mathrm{~mL}$ of PVP (147 mM) was injected into the flask followed by the addition of $7.5 \mathrm{~mL}$ of $\mathrm{AgNO}_{3}(94 \mathrm{mM})$ with a syringe pump at a rate of $0.25 \mathrm{~mL} / \mathrm{min}$. The reaction was allowed to proceed till grey wispy precipitates were observed. Upon the formation of the precipitates, reaction was quenched in an ice-water bath. The product were then washed with ethanol four times and stored in ethanol till further use.

\section{Synthesis of Thick Ag Nanowires}

For a typical synthesis, $10 \mathrm{~mL}$ of ethylene glycol was added to a $100 \mathrm{~mL}$ round bottom flask with a stir bar added. The round bottom flask was then suspended in an oil bath with thermostat set at $151{ }^{\circ} \mathrm{C}$ and heating for $1 \mathrm{~h}$ under magnetic stirring $(250 \mathrm{rpm})$. All reagents were dissolved in ethylene glycol. Then, $80 \mu \mathrm{L} \mathrm{of} \mathrm{CuCl}_{2} .2 \mathrm{H}_{2} \mathrm{O}(4 \mathrm{mM})$ was added into the flask and allowed to heat for another $15 \mathrm{~min} .3 \mathrm{~mL}$ of PVP (147 mM) was injected into the flask followed by the addition of $3 \mathrm{~mL}$ of $\mathrm{AgNO}_{3}(94 \mathrm{mM})$ with a syringe pump at a rate of $0.1 \mathrm{~mL} / \mathrm{min}$. The reaction was allowed to proceed till grey wispy precipitates were observed. Upon the formation of the precipitates, $7.5 \mathrm{~mL}$ of $\mathrm{AgNO}_{3}(94 \mathrm{mM})$ and $7.5 \mathrm{~mL}$ of PVP $(147 \mathrm{mM})$ was added simultaneously at a rate of $(0.05 \mathrm{~mL} / \mathrm{min})$. Reaction was quenched 30 min after the addition in an ice-water bath. The product were then washed with ethanol four times and stored in ethanol till further use.

\section{Attaching Ag Nanowire on Si Wafer}

Si wafer were diced into $4 \mathrm{~mm} \times 4 \mathrm{~mm}$ pieces and soaked in ethanol with sonication for $10 \mathrm{~min}$. Then, they were blow dried with nitrogen gas and oxygen plasma for 10 min. During oxygen plasma, APTES solution $(2 \mu \mathrm{L} / \mathrm{mL})$ in DI water and ethanol mixture with ratio 1:1 was prepared. Si wafers were placed in the APTES solution and allowed to incubate at room temperature for $1 \mathrm{~h}$. The wafers were removed from the solution, washed with ethanol thoroughly and blow dried with nitrogen gas. Ag nanowires were transferred from ethanol solution to DI water and diluted to desired concentration. $20 \mu \mathrm{L}$ of Ag nanowires in DI water was carefully placed on top of the wafer and incubated at 
room temperature for $1 \mathrm{~h}$ to allow Ag nanowires to adhere onto the APTES pretreated Si wafers. The wafers were then washed with DI water to remove any excess Ag nanowires that were not adhere to the surface of the Si wafer. Then, the Si wafers with nanowires were left to dry in air before further use.

\section{Other Substrates for Etching}

Quartz, $\mathrm{Al}_{2} \mathrm{O}_{3}$ and polydimethylsiloxane (PDMS) was also used as substrates for etching. The nanowires were also attached onto these substrates following the above method.

\section{Etching of Ag Nanowires}

Ag nanowires on Si wafer were carefully placed in a beaker which was preheated in an oil bath at $80{ }^{\circ} \mathrm{C}, 20 \mu \mathrm{L}$ of $\mathrm{AgNO}_{3}(94 \mathrm{mM})$ in ethylene glycol was carefully placed on top of the Si wafer and heated for $20 \mathrm{~min}$ in the beaker. The Si wafer with nanowires was carefully removed, washed in DI water to remove excess reactants and dried in air. The etching procedure was repeated for 5 times when the nanowires used were thin. For the thick nanowires and mixture of thin and thick nanowires, the etching procedure was repeated for 10 times.

\section{Controlled Experiments for the Etching of Ag Nanowires}

$100 \mathrm{mM}$ of $\mathrm{CF}_{3} \mathrm{COOAg}, \mathrm{HNO}_{3}, \mathrm{H}_{2} \mathrm{SO}_{4}, \mathrm{HCl}, \mathrm{H}_{3} \mathrm{PO}_{4}, \mathrm{CF}_{3} \mathrm{COOH}$, and $\mathrm{NaNO}_{3}$ in ethylene glycol were prepared as reaction mixtures. Then, the etching-washing cycles were performed as above with these reaction mixtures. 

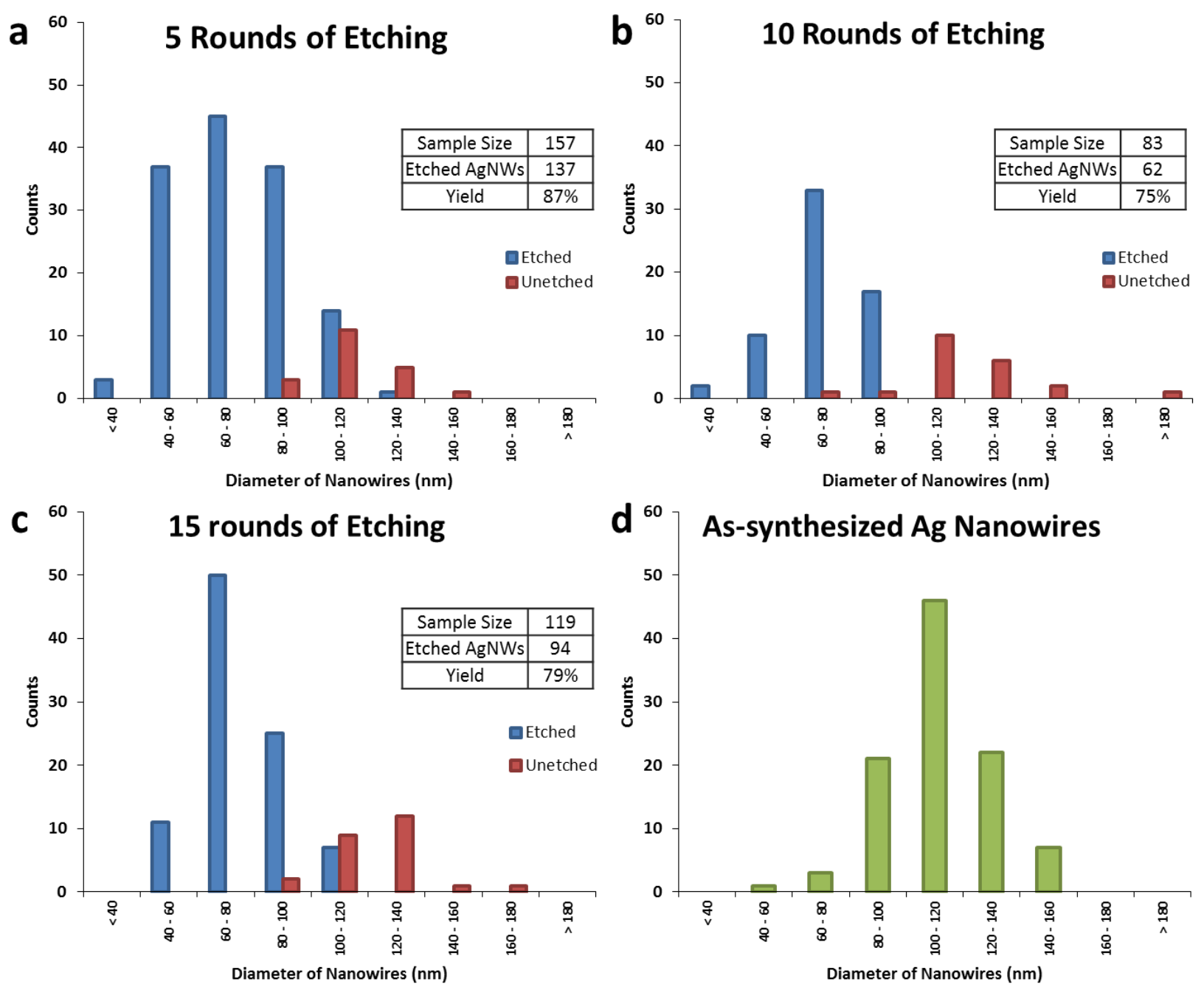

Figure S1. Histograms showing the diameters of etched and non-etched Ag nanowires in samples which undergone (a) 5 rounds, (b) 10 rounds, and (c) 15 rounds of etching-washing. The percentage yield was also tabulated for the following samples. (d) Histogram showing the distribution of nanowire diameters before etching. It appears that the thinner nanowires (below $60 \mathrm{~nm}$ ) were etched away with additional steps. 

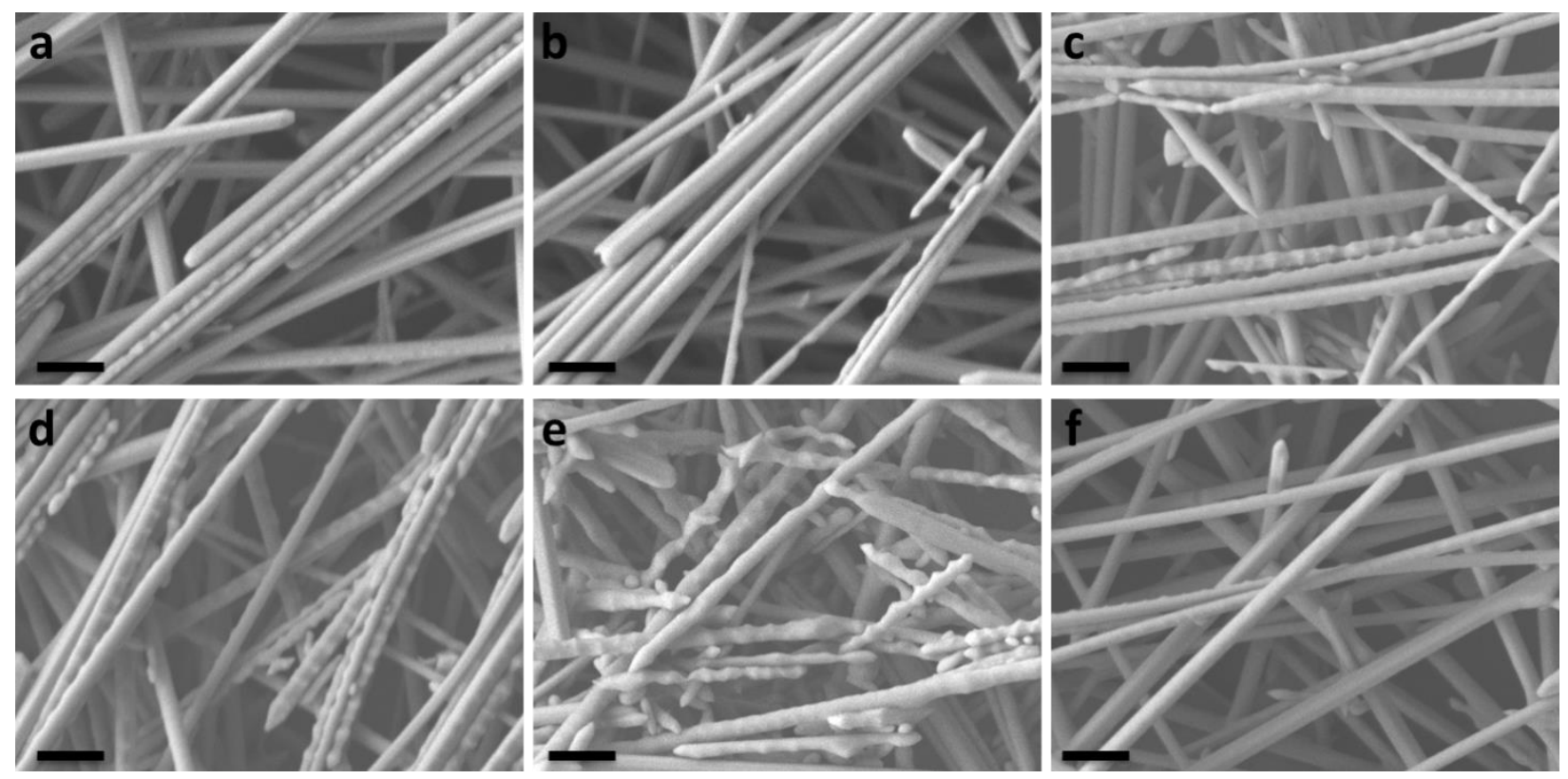

Figure S2. SEM images of Ag nanowires after etching (a) 1 round, (b) 2 rounds, (c) 3 rounds, (d) 4 rounds, (e) 5 rounds with $100 \mathrm{mM}$ of $\mathrm{AgNO}_{3}$ in ethylene glycol at $80{ }^{\circ} \mathrm{C}$ for $20 \mathrm{~min}$ and (f) 1 round of etching with $100 \mathrm{mM}$ of $\mathrm{AgNO}_{3}$ in ethylene glycol at $80^{\circ} \mathrm{C}$ for $100 \mathrm{~min}$. Scale bars are $500 \mathrm{~nm}$. 

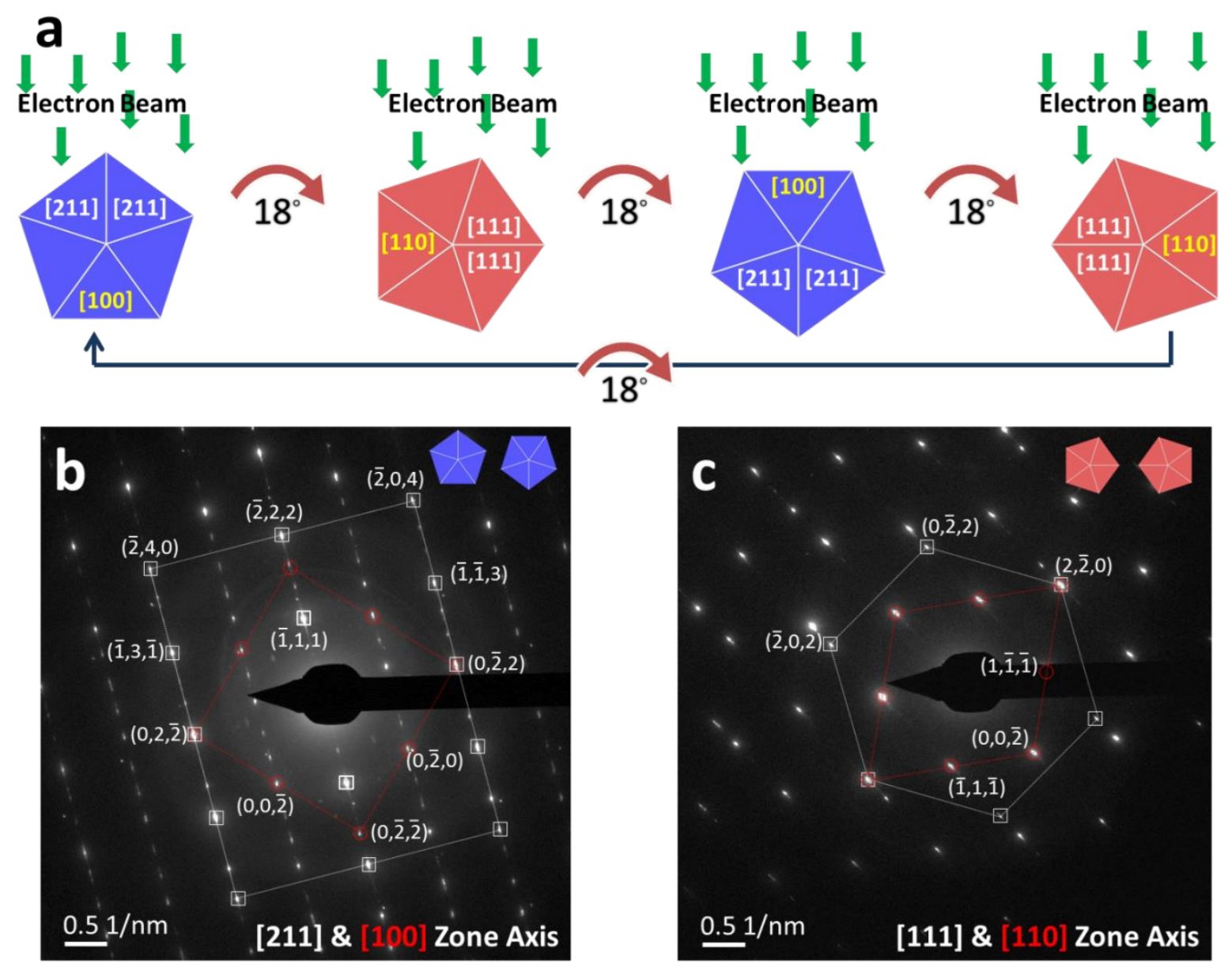

Figure S3. (a) Schematic illustrating the different orientations of penta-twinned nanowires with respect to the electron beam with their zone axis labeled. Overlapped diffraction pattern of (b) [211] and [100] zone axes and (c) [111] and [110] zone axes. 


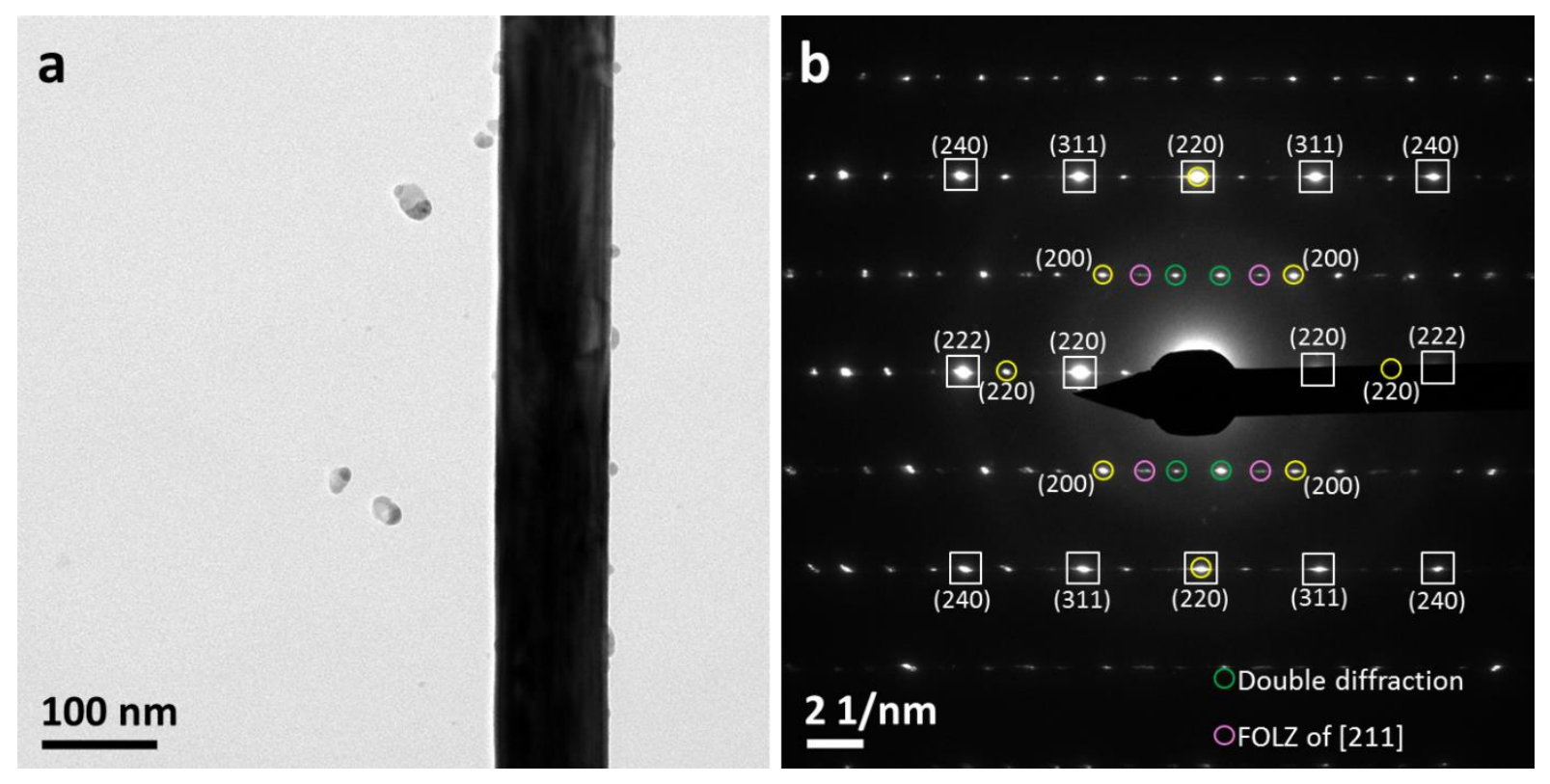

Figure S4. (a) TEM image of an as-synthesized Ag nanowire (Figure 2b) and its corresponding (b) diffraction pattern.

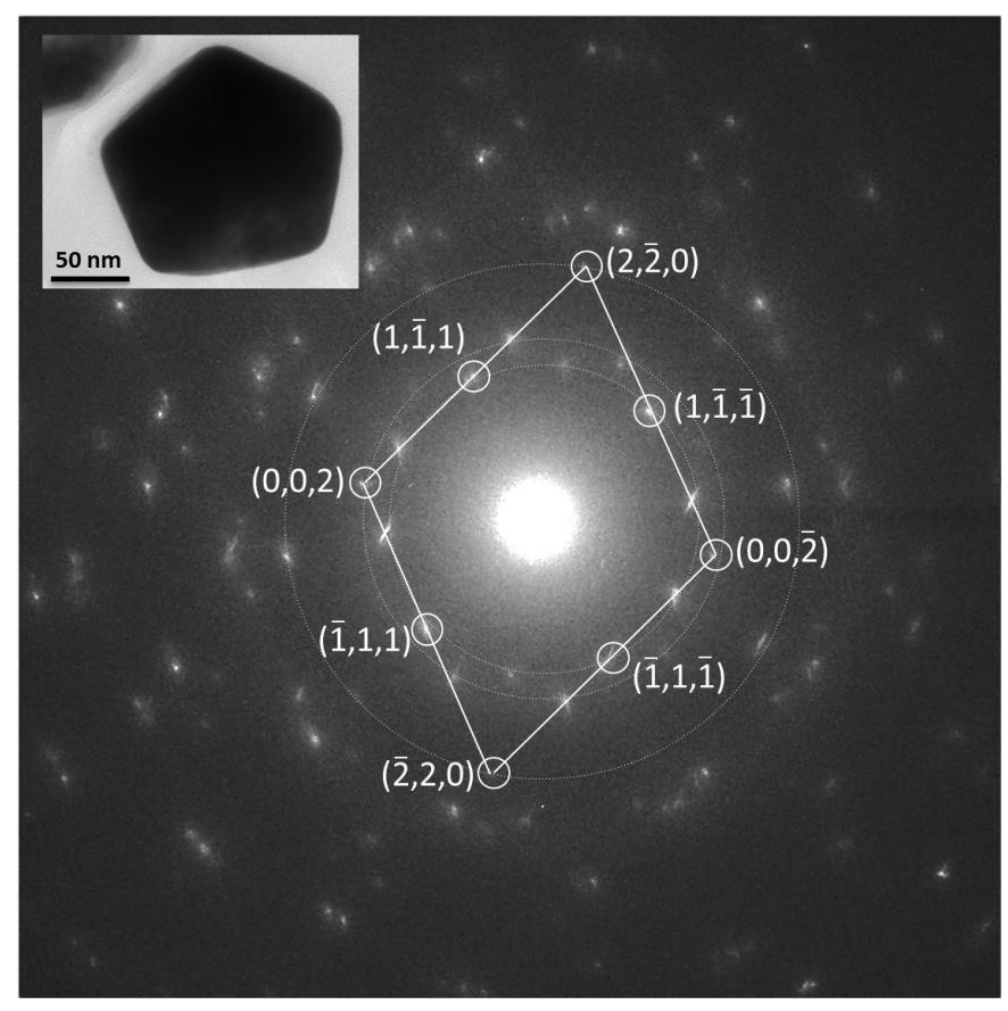

Figure S5. Diffraction pattern of a cross sectioned Ag nanowire (Figure 2c). Inset is the TEM image of the nanowire. 

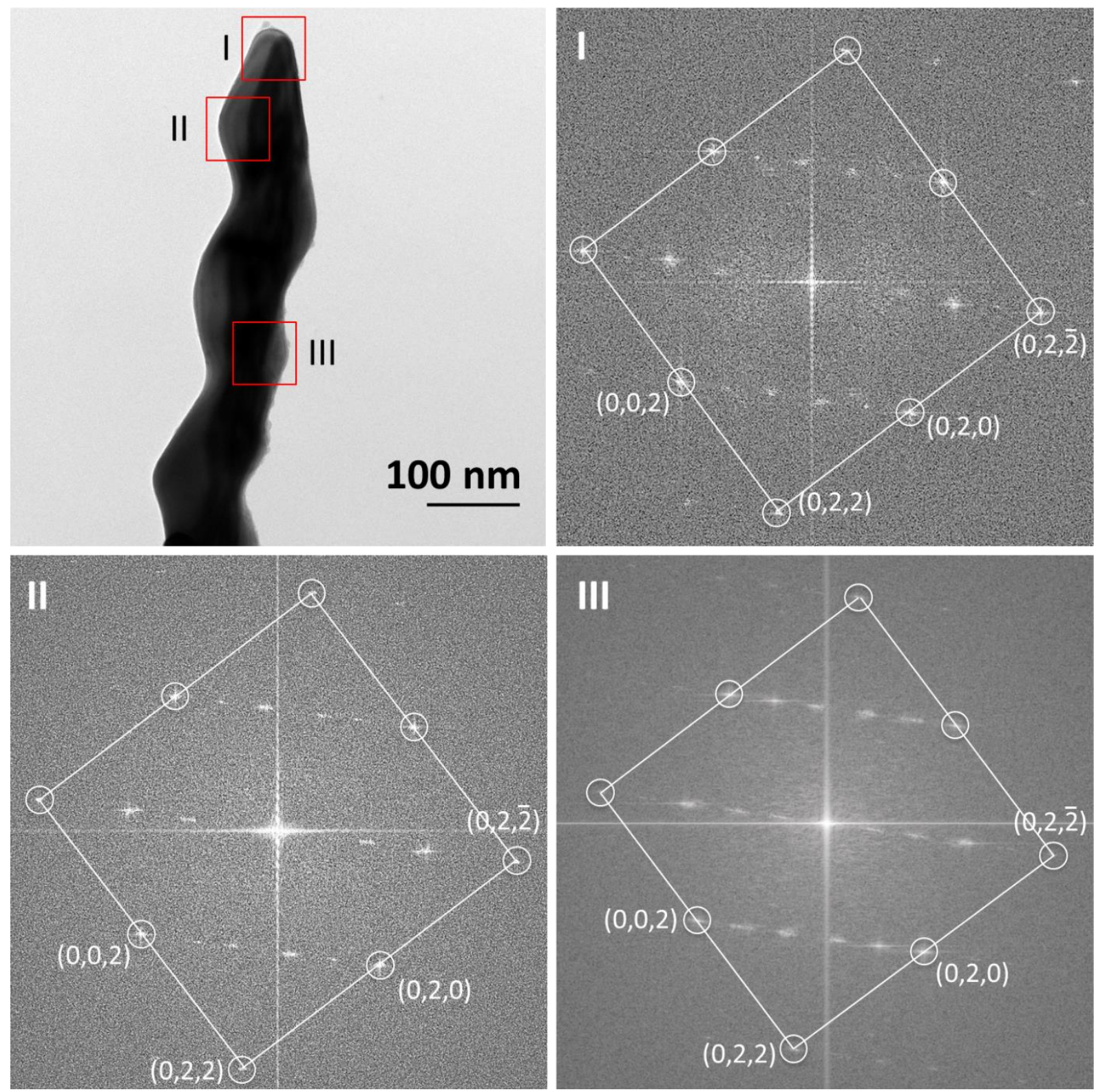

Figure S6. TEM image of an etched Ag nanowire (Figure 2d) and FFT images from different regions of the nanowire. 


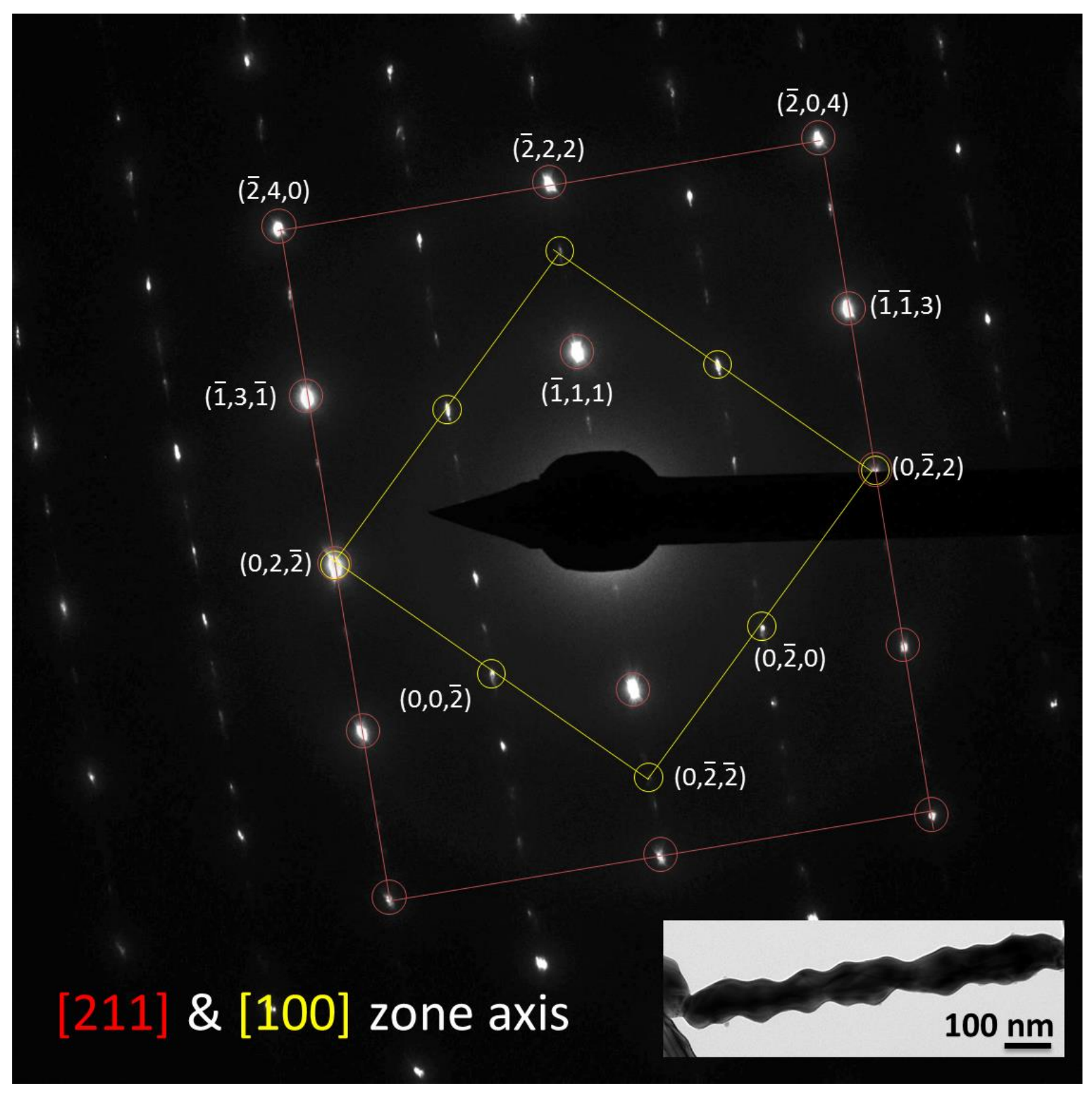

Figure S7. Diffraction pattern of an etched nanowire. Inset is the TEM image of the nanowire. 

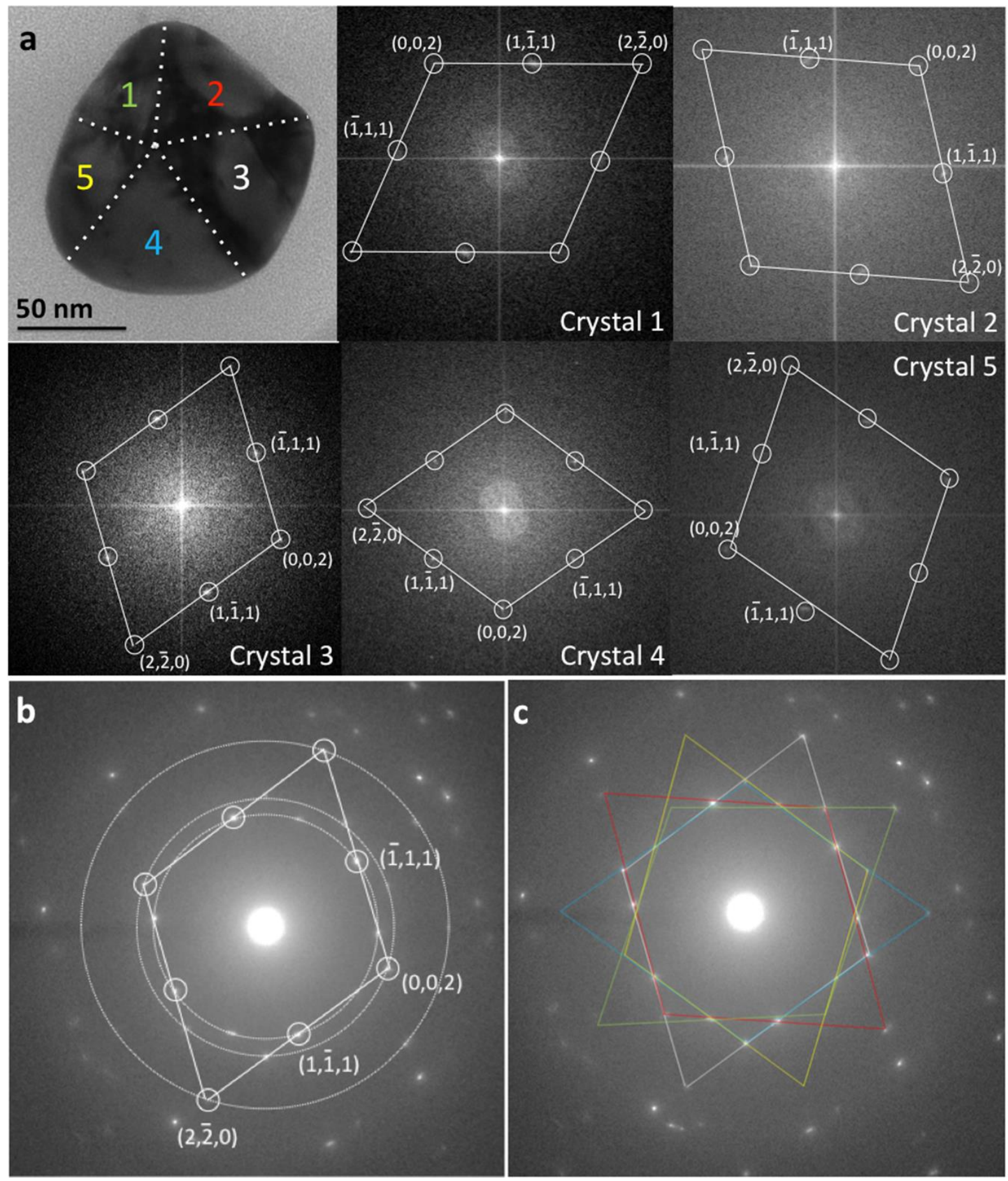

Figure S8. (a) TEM image of a cross sectioned etched Ag nanowire (Figure 2e) and FFT of all five crystals. Diffraction pattern of the same cross sectioned etched Ag nanowire (b) with one crystal labelled and (c) pattern of all five crystals overlapped. 

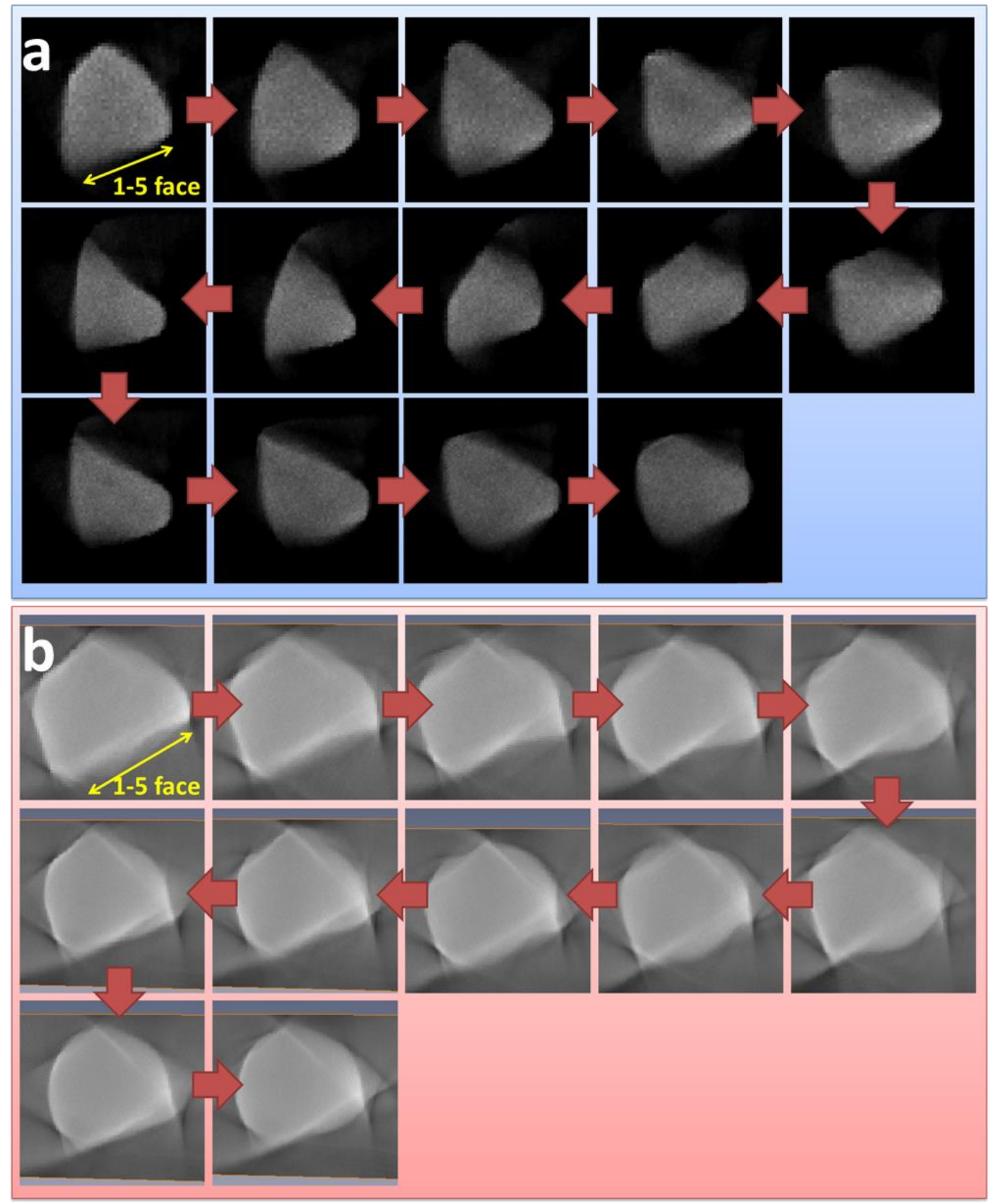

Figure S9. Cross sections obtained from different regions of 3D tomography models: (a) thin helical nanowires and (b) thick nanowires after etching. As the thickness of microtome slices are approximately 50-70 nm thick, we are not able to observe some of the above cross section on TEM analysis. 


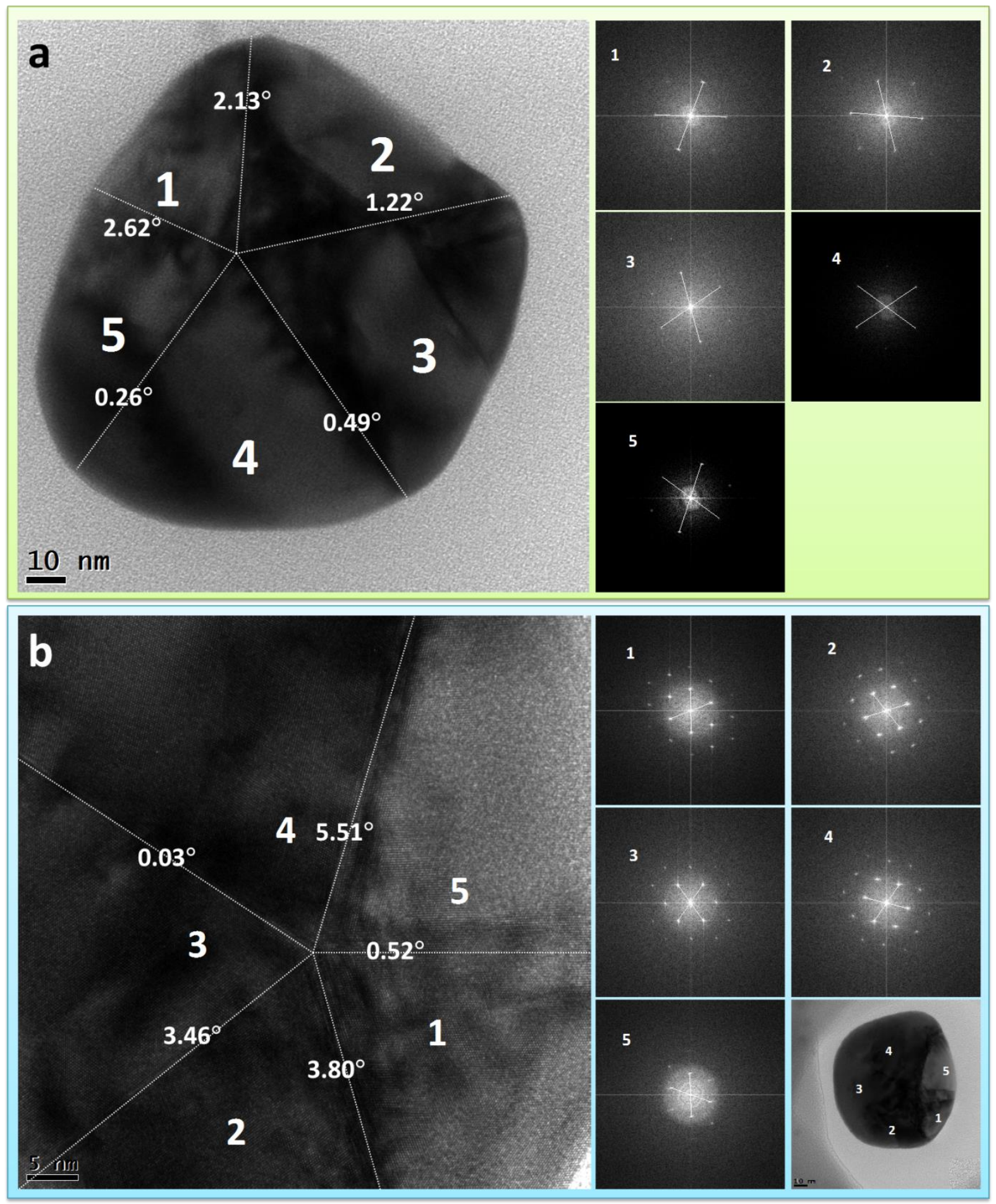

Figure S10. (a,b) Measurements of angles between $\{111\}$ facets of adjacent crystals to determine the misfit angles in each twin boundary. The angle at twinned boundary between Crystal 1 and 5 does not show a larger composition of the $7.35^{\circ}$ angle. Due to imperfection of the pentagonal structure, the angles do not add up to $7.35^{\circ}$. 


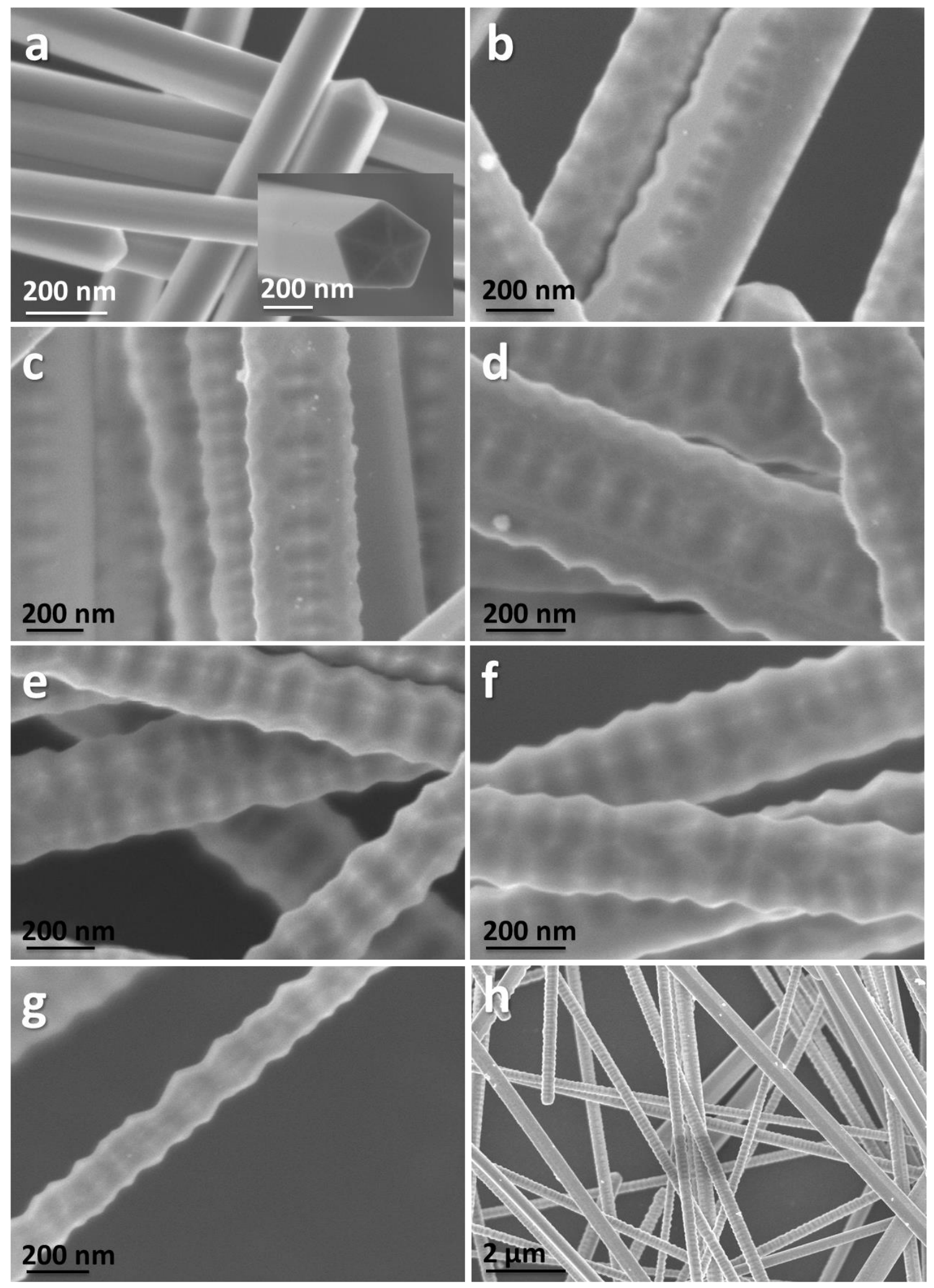

Figure S11. SEM images of thick nanowires (a) before and (b-h) after etching. 

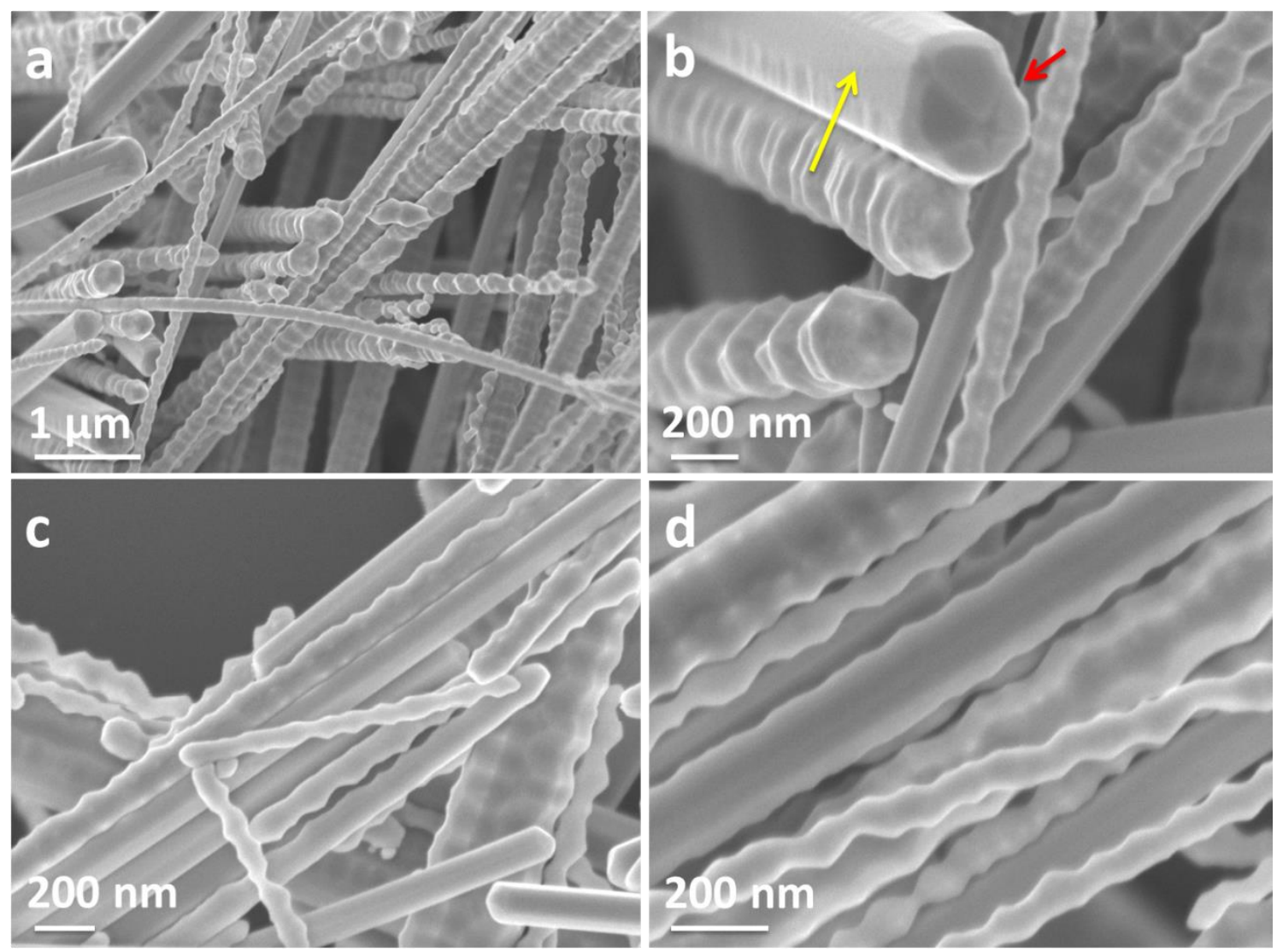

Figure S12. (a,b,c,d) SEM images of thick and thin nanowires mixed into a single sample and etched. Yellow arrow shows the remaining $\{100\}$ facet and the red one shows the more extensively etched twinned boundary. 


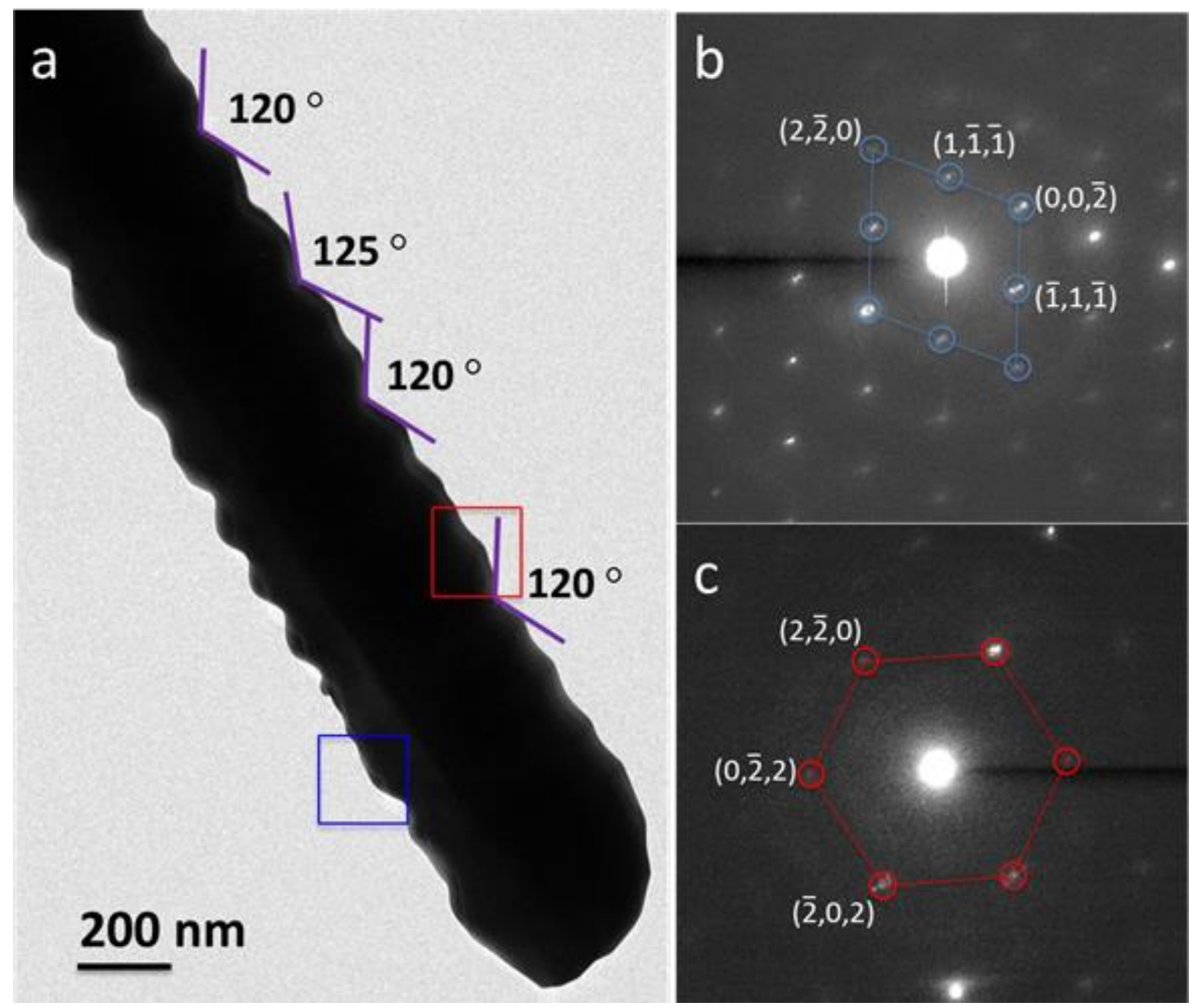

Figure S13. (a) TEM image of thick nanowires used for 3D tomography (Figure 3d-m) and (b,c) its corresponding diffraction pattern at different regions in (a). 

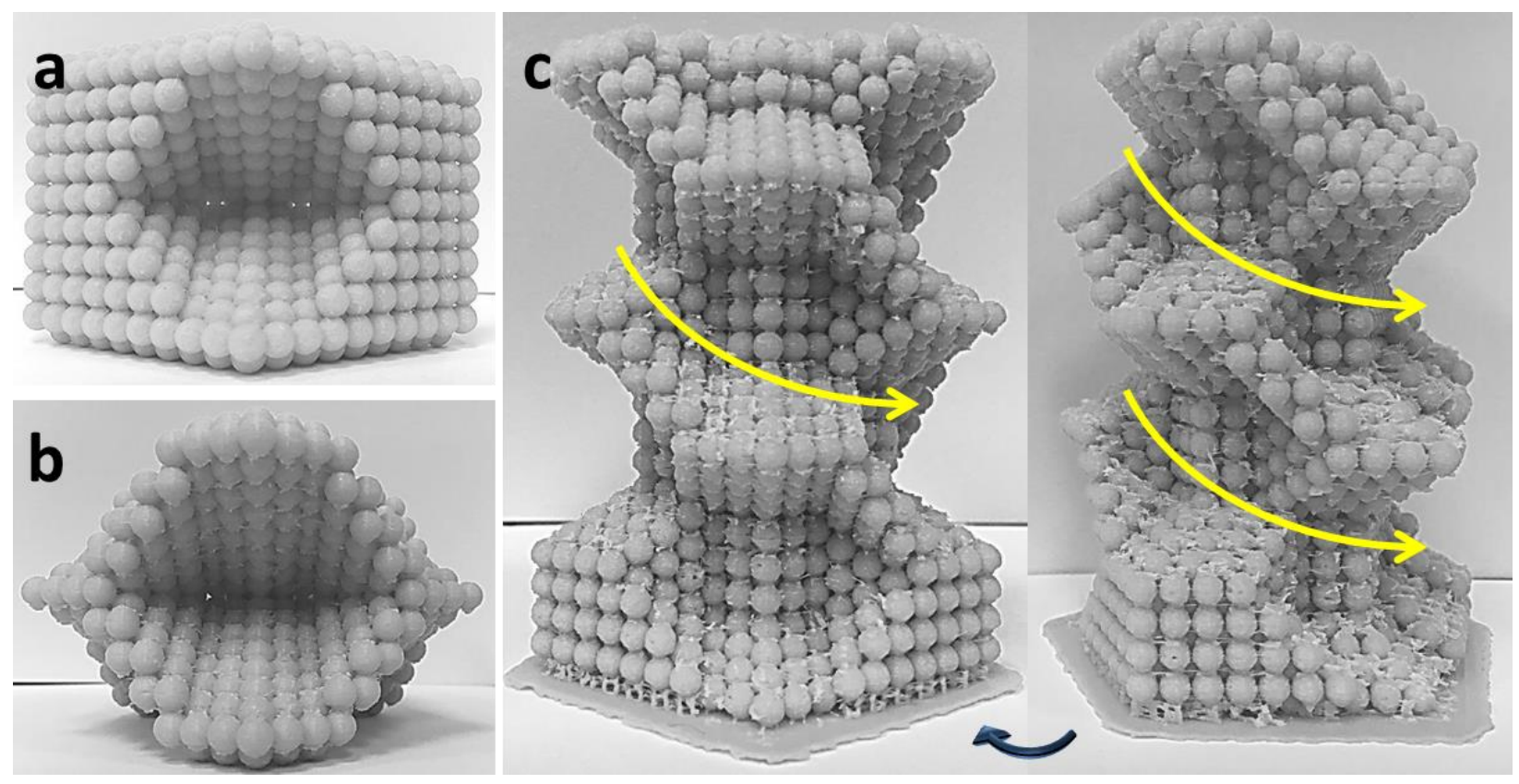

Figure S14. 3D models showing the (a) etch pit on a twinned boundaries, (b) adjacent pits colliding into each other and (c) breaking of "walls" between the pits. 

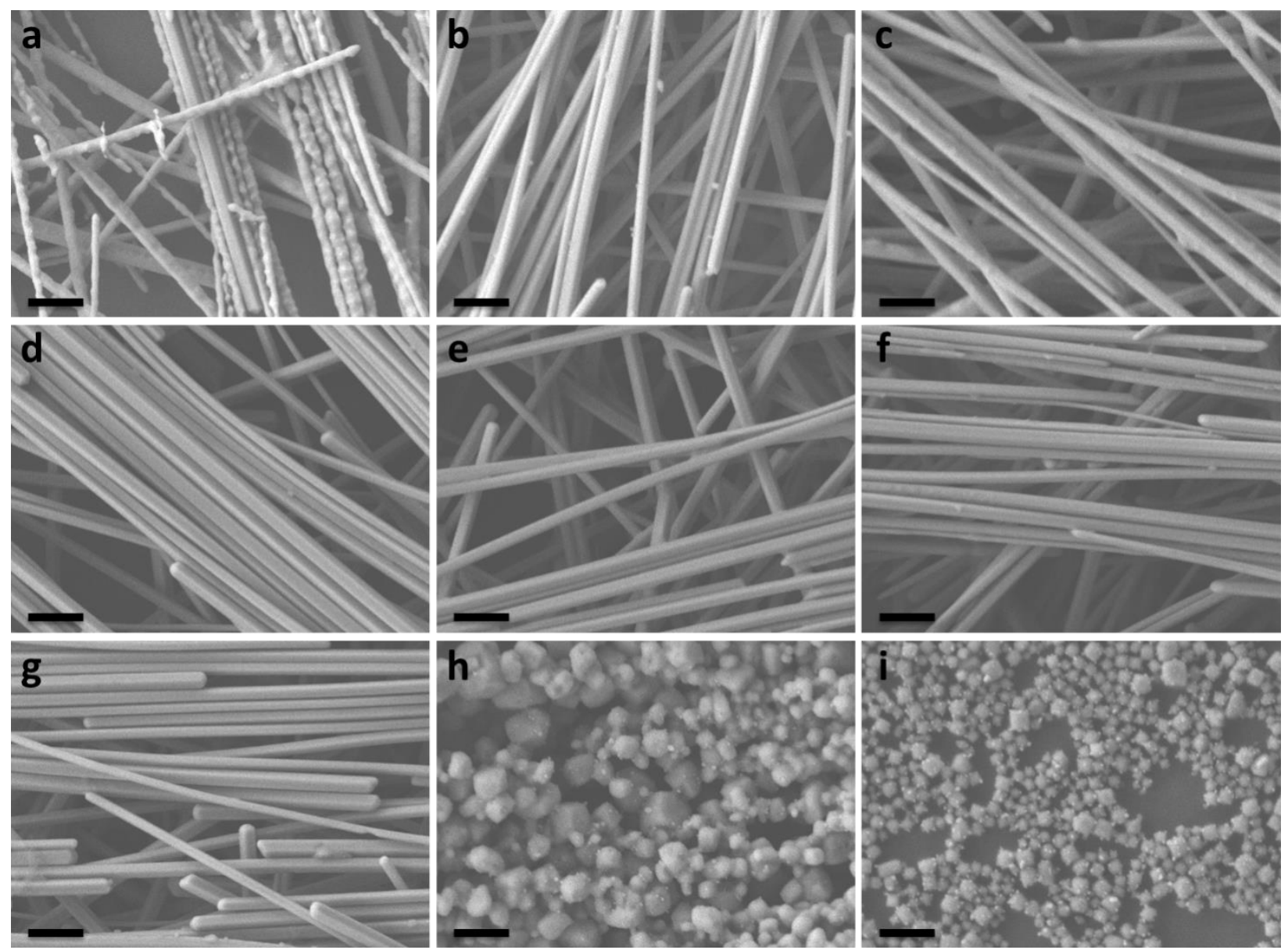

Figure S15. SEM images of $\mathrm{Ag}$ nanowires after five rounds of etching at $80{ }^{\circ} \mathrm{C}$ for 20 min with $100 \mathrm{mM}$ of (a) $\mathrm{CF}_{3} \mathrm{COOAg}$, (b) $\mathrm{HNO}_{3}$, (c) $\mathrm{H}_{2} \mathrm{SO}_{4}$, (d) $\mathrm{HCl}$, (e) $\mathrm{H}_{3} \mathrm{PO}_{4}$, (f) $\mathrm{CF}_{3} \mathrm{COOH}$, and (g) $\mathrm{NaNO}_{3}$. SEM images of Ag nanowires after etching for one round with $100 \mathrm{mM}$ of (h) $\mathrm{FeCl}_{3}$, (i) $\mathrm{Fe}\left(\mathrm{NO}_{3}\right)_{3}$ at $80{ }^{\circ} \mathrm{C}$ for $20 \mathrm{~min}$. Scale bars are 500 nm.
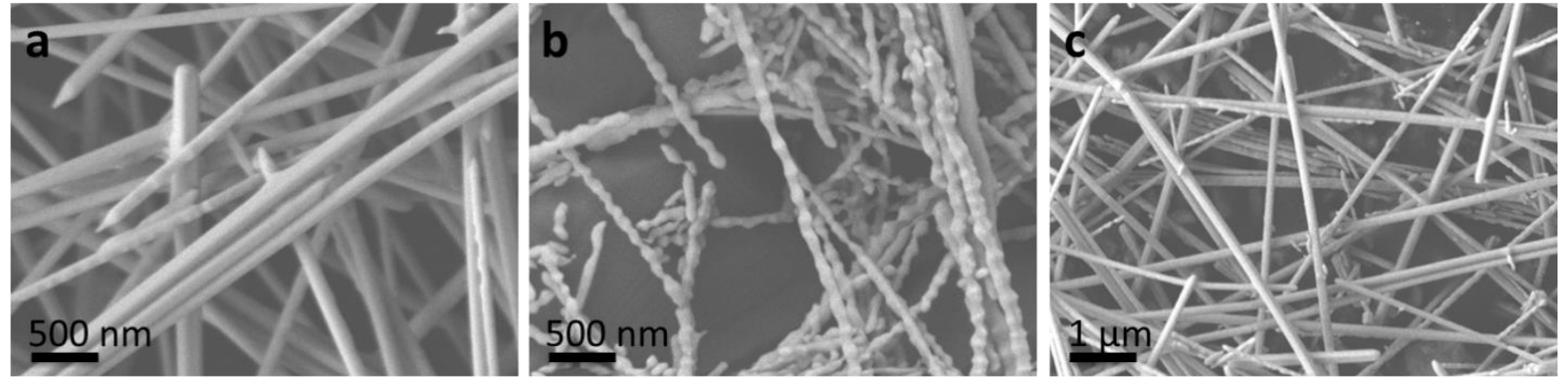

Figure S16. SEM images of $\mathrm{Ag}$ nanowires after etching 5 rounds with $100 \mathrm{mM}$ of $\mathrm{AgNO}_{3}$ in ethylene glycol at 80 ${ }^{\circ} \mathrm{C}$ for $20 \mathrm{~min}$ on (a) $700 \mu \mathrm{m}$ quartz, (b) $500 \mu \mathrm{m} \mathrm{Al}{ }_{2} \mathrm{O}_{3}$ and (c) $1 \mathrm{~mm}$ polydimethylsiloxane (PDMS) substrates. The thicknesses of the substrates are not the same, affecting the heat transfer from the heating source to the nanowires. 

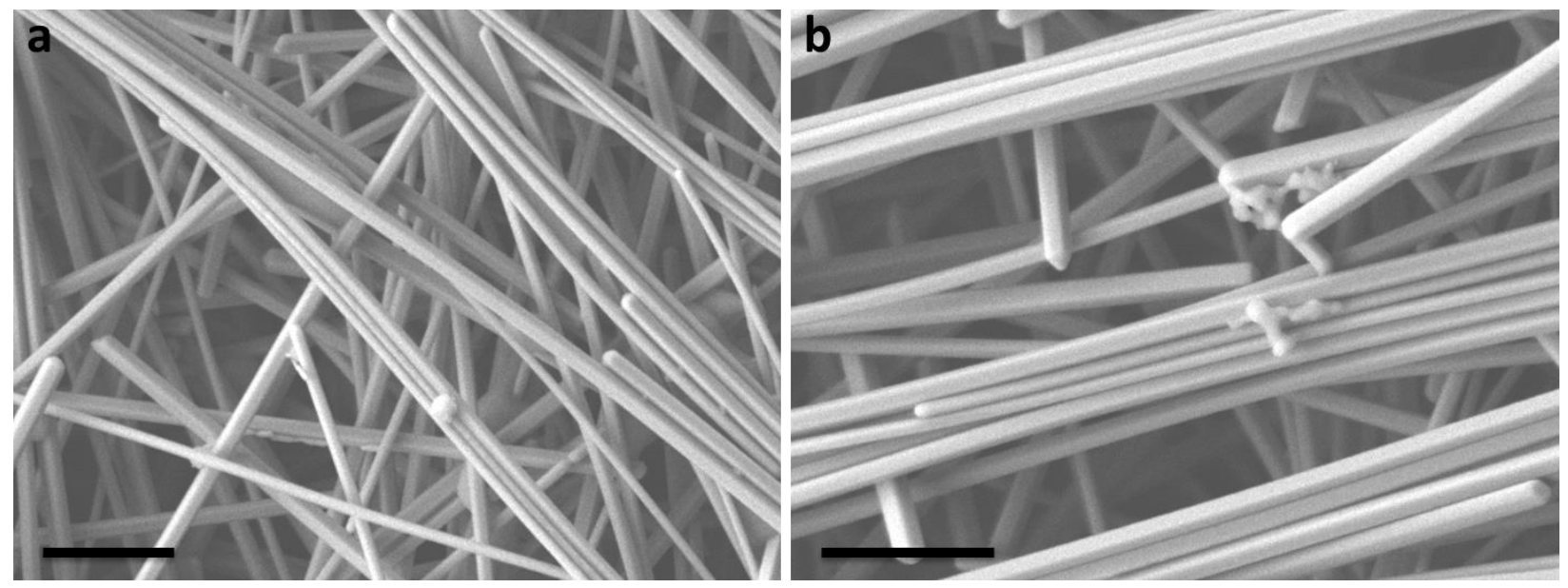

Figure S17. SEM images of Ag nanowires after etching (a) 5 rounds with $100 \mathrm{mM} \mathrm{AgNO}_{3}$ in 1,5-pentanediol and (b) 1 round with $100 \mathrm{mM} \mathrm{AgNO}_{3}$ in dimethylformamide (DMF). Scale bars are $1 \mu \mathrm{m}$. 